\title{
The unmet need for treatment of children with musculoskeletal impairment in Malawi
}

\author{
Leonard Banza Ngoie 1,2,3* Eva Dybvik4 , Geir Hallan²,5, Jan-Erik Gjertsen 2,6, Nyengo Mkandawire ${ }^{7,8}$, \\ Carlos Varela ${ }^{1,2,7}$ and Sven Young ${ }^{1,6,7}$
}

\begin{abstract}
Background: More than a billion people globally are living with disability and the prevalence is likely to increase rapidly in the coming years in low- and middle-income countries (LMICs). The vast majority of those living with disability are children residing in LMICs. There is very little reliable data on the epidemiology of musculoskeletal impairments (MSIs) in children and even less is available for Malawi. Previous studies in Malawi on childhood disability and the impact of musculoskeletal impairment (MSI) on the lives of children have been done but on a small scale and have not used disability measurement tools designed for children. Therefore in this study, we aimed to estimate the MSI prevalence, causes, and the treatment need among children aged 16 years or less in Malawi.

Methods: This study was carried out as a national cross sectional survey. Clusters were selected across the whole country through probability proportional to size sampling with an urban/rural and demographic split that matched the national distribution of the population. Clusters were distributed around all 27-mainland districts of Malawi. Population of Malawi was 18.3 million from 2018 estimates, based on age categories we estimated that about 8.9 million were 16 years and younger. MSI diagnosis from our randomized sample was extrapolated to the population of Malawi, confidence limits was calculated using normal approximation.
\end{abstract}

Results: Of 3792 children aged 16 or less who were enumerated, 3648 (96.2\%) were examined and 236 were confirmed to have MSI, giving a prevalence of MSI of 6.5\% (CI 5.7-7.3). Extrapolated to the Malawian population this means as many as 576,000 (95\% Cl 505,000-647,000) children could be living with MSI in Malawi. Overall, 46\% of MSIs were due to congenital causes, 34\% were neurological in origin, $8.4 \%$ were due to trauma, $7.8 \%$ were acquired non-traumatic non-infective causes, and $3.4 \%$ were due to infection. We estimated a total number of $112,000(80,000-$ $145,000)$ children in need of Prostheses and Orthoses (P\&O), 42,000 $(22,000-61,000)$ in need of mobility aids (including 37,000 wheel chairs), 73,000 (47,000-99,000) in need of medication, 59,000 (35,000-82,000) in need of physical therapy, and 20,000 (6000-33,000) children in need of orthopaedic surgery. Low parents' educational level was one factor associated with an increased risk of MSI.

Conclusion: This survey has uncovered a large burden of MSI among children aged 16 and under in Malawi. The burden of musculoskeletal impairment in Malawi is mostly unattended, revealing a need to scale up both P\&O services, physical \& occupational therapy, and surgical services in the country.

Keywords: Musculoskeletal impairment, Childhood disability, Cluster randomized survey, Malawi

*Correspondence: leonardbanza2014@gmail.com

${ }^{1}$ Department of Surgery, Kamuzu Central Hospital, P.O. Box 149, Lilongwe, Malawi

Full list of author information is available at the end of the article

\section{Introduction}

More than a billion people globally are living with disability [1], and the prevalence is likely to increase rapidly in the coming years in low- and middle-income countries 
(LMICs) [2, 3]. The vast majority of those living with disability are children residing in LMICs [4]. There is increasing evidence that children with disabilities are more likely to come from poorer households, are substantially less likely to attend school and experience poorer health compared to their non-disabled peers $[2,5]$. Fewer than $10 \%$ of children with disabilities are estimated to attend school in Africa [3]. Disability is argued to have a greater impact on access to education than gender, economic status, or rural/urban residence, and is associated with longterm poverty [6].

In 2003, the WHO published a report highlighting the burden of musculoskeletal conditions in low resource countries [7]. In Africa, conservative estimates state that $11 \%$ of the total burden of disease, and 25 million disability-adjusted life years (DALYs) (38/1000 population), are due to surgical conditions [8]. There is very litthe reliable data on the epidemiology of musculoskeletal impairments (MSIs) in children or the consequences of disability on people with MSI in LMICs [1], and even less is available for Malawi.

A national survey in Rwanda estimated that 2.58\% of children under 16 year old have one or more MSIs, of which $43 \%$ are moderate or severe [9]. There have been studies in Malawi on childhood disability [10] and impact of musculoskeletal impairment (MSI) in the lives of children [11]. However, these surveys were done on a small scale and did not use disability measurement tools designed for children. Also, there was no verification of self-reported functional limitations by clinical examination.

There is an urgent need to plan appropriate and accessible services for children with MSI in Malawi, and for evidence-based advocacy for funding of this. The authors carried out a national cluster-randomized household survey in 2016, revealing an overall prevalence of MSI of 9.5\% (CI 8.9-10.1) in the Malawian population [12]. Using data from this survey, we aimed to estimate the prevalence and the treatment need among children aged 16 years or less in Malawi, and to describe the causes of MSI in Malawian children.

\section{Methods}

This study was based on a cross sectional survey. A list of enumeration areas from the Malawi Census Board for the 2008 national census records was received from the National Statistic Office. Clusters were selected across the whole country through probability proportional to size sampling with an urban/rural and demographic split that matched the national distribution of the population. Clusters were distributed around all 27 mainland districts of Malawi, many at very remote locations with poor roads, and it took the team 2 months, from 1st July to 30th August 2016, to reach all the selected villages by car. Depending on the size of the village, two to four households were randomly selected by spinning a bottle. All individuals present were examined in their households by survey field teams. The standardized selection, interview and examination protocol has been described in detail previously [13] but is outlined here. The definition of MSI that was used was developed from the WHO International Classification of Function (ICF) [14]:

“... . a lack of normal structure or function, or an increase in pain or discomfort in the integument, muscles, bone or joints of the body of an individual, that has lasted at least 1 month and which limits function of the musculoskeletal system ... "

Data collection was done by 32 third-year medical students. They all underwent a 14 days training supervised by two orthopaedic surgeons and two senior orthopaedic clinical officers on how to assess persons with musculoskeletal impairment and the use of the questionnaire and computer tablet. All participants were screened for MSI by asking them seven questions about difficulties using their musculoskeletal system and how long they have had these symptoms. The age cut off of 16 years and under was chosen for ease of comparison to a similar study done in Rwanda [9]. Also, children aged 17 and over are expected to be past or near skeletal bone maturity. According to cultural definitions of adulthood, the latter are usually considered as young adults in our setting.

The screening tool called "Rapid Assessment of Musculoskeletal Impairment" (see Appendix) was developed by researchers and clinical staff at the University of Oxford and the London School of Hygiene and Tropical Medicine for a previous survey in Rwanda, and has been shown to have $99 \%$ sensitivity and $97 \%$ specificity with interobserver Kappa scores of 0.90 for the diagnostic group [15]. For the youngest (age below 5) household members, the guardian of the child was interviewed. For all participants we also did screen parents' educational level.

The standardized examination protocol used, if screening questions revealed any MSI, comprised the elements seen in Table 1:

All children identified as having impairment in the study were referred to field workers of a communitybased rehabilitation (CBR) program for appropriate action such as physiotherapy, prosthetic and orthotic $(\mathrm{P} \& \mathrm{O})$ devices, or mobility aids. Children found in need of surgery (indication made by the orthopaedic surgeon on the survey team) were referred to a specialist facility for treatment.

Categorical variables was analyzed using a Pearson's chi square test, continuous variable was tested using 
Table 1 Standardized interview and examination protocol

\begin{tabular}{|c|c|}
\hline Elements & Definition \\
\hline Diagnosis & $\begin{array}{l}\text { Diagnosis categorized as: neurological, traumatic, congenital, metabolic, infective, or acquired non- } \\
\text { traumatic non-infective. Within these categories an algorithm was created and used to give a specific } \\
\text { diagnosis. Up to two diagnoses were permissible per each identified case of MSI [15]. }\end{array}$ \\
\hline Severity & $\begin{array}{l}\text { Severity was determined using ICF parameters for the amount of function which was lost through } \\
\text { the presence of the impairment. This was classified as "mild", "moderate" or "severe" [13]. Severity was } \\
\text { determined using the parameters for the percentage of function outlined in the WHO reference book } \\
\text { International Classification of Functioning (ICF) [14]. A loss of function of 5-24\% was mild, 25-49\% was } \\
\text { moderate and 50-90\% was severe }\end{array}$ \\
\hline Treatment received & Any known treatment given to the participant (medical or other) was recorded \\
\hline Treatment needed & Treatment required by the participants was assessed according to Malawi standard treatment guideline \\
\hline
\end{tabular}

independent t-test. The population of Malawi was 18.3 million according to 2018 estimates, and based on the age categories in the same census, we estimated that about 8.9 million were 16 years and younger [12]. MSI diagnosis from our randomized sample was extrapolated to the population of Malawi, confidence limits were calculated using normal approximation. The statistical analyses were performed in the statistical package IBM SPSS Statistics Version 26 (IBM Corp., Armonk, NY, USA) and the statistical package R (http://CRAN.R-project.org). P-values less than 0.05 was considered statistically significant.

The approval to conduct the survey was granted by the University of Malawi College of Medicine Research and Ethics Committee (COMREC) and The Regional Committee for Medical and Health Research Ethics (REC West) in Norway. Data collection and handling followed national guidelines in line with the Declaration of Helsinki. Consent to survey the districts and clusters were granted respectively by the District Commissioner and village head for each visited district and cluster. Informed consent to participate in the study was obtained from a parent or guardian before examination. No names of participants, or locations of surveyed households within a cluster were registered in the survey data. Data collectors were allowed to take clinical photographs for teaching and discussion of follow up after a verbal informed consent was granted from the parent or guardian. If a child was found to have a condition needing treatment, a referral to the correct institution was made by the first author, and data kept separate from, and with no linkage to, the study data.

\section{Results}

Of 3792 children aged 16 or less who were enumerated, $3648(96.2 \%)$ were examined. $3.8 \%$ of children were not available during the first screening or at one more subsequent visit to the household. Of the 3648 examined participants, 236 were confirmed to have MSI, giving a prevalence of MSI in the 16 and under age group of $6.5 \%$
(CI 5.7-7.3). Extrapolated to the Malawian population this means that as many as 576,000 (95\% CI 505,000$647,000)$ children could be living with MSI in Malawi.

Among children found to have MSI, there was no significant difference in the prevalence between children aged $5-16$ and those under 5 years of age $(p=0.56)$. Boys were found to have marginally more MSI than girls (7\% vs $6 \%)(p=0.046)$.

Using the ICF classification the MSIs were classified as moderate or severe in more than 3 out of 4 of the children with MSI. Low parents' educational level was associated with more MSI among the family members (Table 2).

A total of 358 diagnoses were registered in the 236 individuals diagnosed with MSI. The diagnoses are summarized in Table 3 with extrapolated numbers of affected children in Malawi. Overall, $46 \%$ of MSIs were due to congenital causes, $34.4 \%$ were neurological in origin, $8.4 \%$ were due to trauma, $7.8 \%$ were acquired nontraumatic non-infective causes and $3.4 \%$ were due to infection.

Of the 236 children diagnosed with MSI, 46 were judged to be in need of prosthetic and orthotic (P\&O) services, 17 in need of mobility aids (including wheelchairs), 30 in need of medication, 24 in need of physical therapy, and 8 in need of orthopaedic surgery. Extrapolating these findings to the population of Malawi, we estimated a total number of $112,000(80,000-145,000)$ children in need of $\mathrm{P} \& \mathrm{O}, 42,000(22,000-61,000)$ in need of mobility aids (including 37,000 wheel chairs), $73,000(47,000-99,000)$ in need of (mainly anti-epileptic) medication, 59,000 (35,000-82,000) in need of physical therapy, and 20,000 (6000-33,000) children in need of orthopaedic surgery (Fig. 1).

\section{Discussion}

This first countrywide survey of MSI in Malawi revealed that $6.5 \%$ of children, or an estimated 576,000 children are living with MSI. Of these, 112,000 children could be in need of $\mathrm{P} \& \mathrm{O}$ devices and 42,000 in need of mobility 
Table 2 Baseline table for the 3648 children

\begin{tabular}{|c|c|c|c|c|}
\hline & Total $(N=3648)$ & MSI $(N=236)$ & No MSI $(N=3412)$ & $p$-value \\
\hline Gender & & & & $0.046^{*}$ \\
\hline Male & 1917 & $137(7 \%)$ & $1780(93 \%)$ & \\
\hline Female & 1731 & $99(6 \%)$ & $1632(94 \%)$ & \\
\hline Age & & & & 0.56 \\
\hline $0-5 y r$ & 1109 & $76(7 \%)$ & $1033(93 \%)$ & \\
\hline $5-16 y r$ & 2539 & $160(6 \%)$ & $2379(94 \%)$ & \\
\hline Location & & & & 0.36 \\
\hline Rural & 3270 & $25(6.5 \%)$ & 3245 (93.5\%) & \\
\hline Urban & 151 & $11(7 \%)$ & $140(93 \%)$ & \\
\hline Missing & 27 & $0(0 \%)$ & $27(100 \%)$ & \\
\hline Parents educational level & & & & 0.005 \\
\hline None & 1073 & $94(9 \%)$ & $979(91 \%)$ & \\
\hline Primary & 2240 & $127(6 \%)$ & $2113(94 \%)$ & \\
\hline Secondary & 260 & $10(4 \%)$ & $250(96 \%)$ & \\
\hline University & 25 & $2(8 \%)$ & $23(92 \%)$ & \\
\hline Missing & 50 & $3(6 \%)$ & $47(94 \%)$ & \\
\hline \multicolumn{5}{|l|}{ Severity MSI** } \\
\hline Mild & 55 & $55(23.3 \%) *$ & 0 & \\
\hline Moderate & 112 & $112(47.5 \%) *$ & 0 & \\
\hline Severe & 69 & $69(29.2 \%) *$ & 0 & \\
\hline Missing & 3412 & 0 & 3412 & \\
\hline
\end{tabular}

*Percent of the $236 \mathrm{MSI}$ cases

${ }^{* *}$ According to the WHO International Classification of Function

aids (including 37,000 in need of wheelchairs) to help with ambulation or activities of daily living; 73,000 need medication; 59,000 could benefit from physical therapy, and approximately 20,000 were estimated to benefit from surgery. Currently it is estimated to be only 14 Prosthetists \& Orthotists, 200 physiotherapists and physiotherapy assistants, and 15 orthopaedic surgeons in the country.

Studies done on MSI in children in Rwanda and in the Fundong District in North-West Cameroon have shown a prevalence of MSI in children of 16 years and younger of 2.58 and $2.9 \%$ respectively $[9,16]$. This is less than half the $6.5 \%$ proportion of children with MSI in Malawi. The reason of this difference remains unclear. However, extrapolated MSI treatment need estimates in our study have shown that approximately 20,000 children would benefit from surgery in Malawi against 50,000 in Rwanda. The reason for this difference could be that diagnoses and surgical indications in our survey were made by a surgeon, in contrast to the studies from Rwanda and Cameroon, where these were made by physiotherapists.

Most MSI diagnoses in our survey were due to congenital deformities $(46 \%)$ or were neurological in nature (34.4\%). There are few studies to compare our numbers with, but a 5-year audit of all elective orthopaedic operations performed in children at a university hospital in Nigeria showed that congenital limb deformities alone accounted for $35.2 \%$ of the diagnoses [17]. A previous study on childhood disability in two districts of Malawi showed that physical impairment (39\%) was the commonest impairment type [10], which is in line with this study.

The neurological conditions identified were predominantly cerebral palsy $(\mathrm{CP})$ and epilepsy, which explains the need of medication and $\mathrm{P} \& \mathrm{O}$ devices in those affected children. $\mathrm{CP}$ is the most common motor disability in children worldwide, with an estimated prevalence of more than 2 per 1000 in high-resource settings, and a higher prevalence of up to 10 per 1000 reported in lowresource settings $[18,19]$. CP is known to be an important and common contributor to childhood disability in low-resource settings [20], as supported by another Malawian study [10].

$47.5 \%$ of children in our survey had moderate MSI, 29.2\% had severe MSI and 23.3\% had mild MSI. These findings support those of the World Bank where recent estimates suggest that $5 \%$ of all children - 93 million children globally - are living with moderate or severe disability as defined by the World Health Organization (WHO) [2]. Our study also showed that low education in the 
Table 3 MSI Diagnosis and Extrapolation to Malawi population

\begin{tabular}{|c|c|c|c|}
\hline Diagnosis & Number & Total in category (\%) & $\begin{array}{l}\text { Extrapolated number of that } \\
\text { diagnosis in Malawi to nearest } 1000 \\
(95 \% \mathrm{Cl})\end{array}$ \\
\hline Congenital deformity & & $165(46 \%)$ & $403,000(343,000-463,000)$ \\
\hline Syndactyly & 13 & & $32,000(15,000-49,000)$ \\
\hline Polydactyly & 29 & & $71,000(45,000-96,000)$ \\
\hline Other UL deformity & 19 & & $46,000(26,000-67,000)$ \\
\hline Club foot & 19 & & $46,000(26,000-67,000)$ \\
\hline Other LL deformity & 28 & & $68,000(43,000-94,000)$ \\
\hline Spine deformity & 43 & & $105,000(74,000-136,000)$ \\
\hline Other congenital deformity & 14 & & $34,000(16,000-52,000)$ \\
\hline Trauma & & $30(8.4 \%)$ & $73,000(47,000-99,000)$ \\
\hline Burn contracture & 6 & & $15,000(3000-26,000)$ \\
\hline Fracture non/ malunion & 7 & & $17,000(4000-30,000)$ \\
\hline Spine injury & 0 & & \\
\hline Head injury & 2 & & $5000(0-12,000)$ \\
\hline Tendon/nerve injury & 6 & & $15,000(3000-26,000)$ \\
\hline Amputation & 5 & & $12,000(2000-23,000)$ \\
\hline Joint chronic dislocation & 4 & & $10,000(0-19,000)$ \\
\hline Other chronic joint injury & 0 & & \\
\hline Neurological & & $123(34.4 \%)$ & $300,000(248,000-353,000)$ \\
\hline Epilepsy & 43 & & $105,000(74,000-136,000)$ \\
\hline Polio (sequelae) & 11 & & $27,000(11,000-43,000)$ \\
\hline Para/quadra/Hemiplegia & 15 & & $37,000(18,000-55,000)$ \\
\hline Cerebral palsy & 46 & & $112,000(80,000-145,000)$ \\
\hline Peripheral nerve palsy & 0 & & \\
\hline Other neurological MSI & 8 & & $20,000(6000-33,000)$ \\
\hline Infective & & $12(3.4 \%)$ & $30,000(13,000-46,000)$ \\
\hline Bone infection limb & 2 & & $5000(0-12,000)$ \\
\hline Joint infection & 3 & & $7000(0-16,000)$ \\
\hline Spine infection & 2 & & $5000(0-12,000)$ \\
\hline Soft tissue infection & 5 & & $12,000(2000-23,000)$ \\
\hline $\begin{array}{l}\text { Other acquired non-infective non- trau- } \\
\text { matic }\end{array}$ & & $28(7.8 \%)$ & $68,000(43,000-94,000)$ \\
\hline Angular limb deformity & 11 & & $27,000(11,000-43,000)$ \\
\hline Joint problem & 3 & & $7000(0-16,000)$ \\
\hline Spine pain & 0 & & \\
\hline Skin/ soft tissue/ bone swelling & 2 & & $5000(0-12,000)$ \\
\hline Limb swelling & 11 & & $27,000(11,000-43,000)$ \\
\hline Limb pain & 0 & & \\
\hline Other acquired spine deformity & 1 & & $403,000(343,000-463,000)$ \\
\hline Total & 358 & & \\
\hline
\end{tabular}

LL Lower limbs, UL Upper Limbs

family was associated with more MSI among the family members. A literature review on disability has suggested that an educated individual with a disability is more likely to better cope with her/his disability than those without education and that chronic health conditions may go improperly monitored by patients who are functionally illiterate and the overall well-being of these individuals may worsen overtime [21].

In common with all population surveys, our study had some limitations. The probability proportional to size sampling, diagnostic tools were limited to history and clinical examination, which restricted the identification 


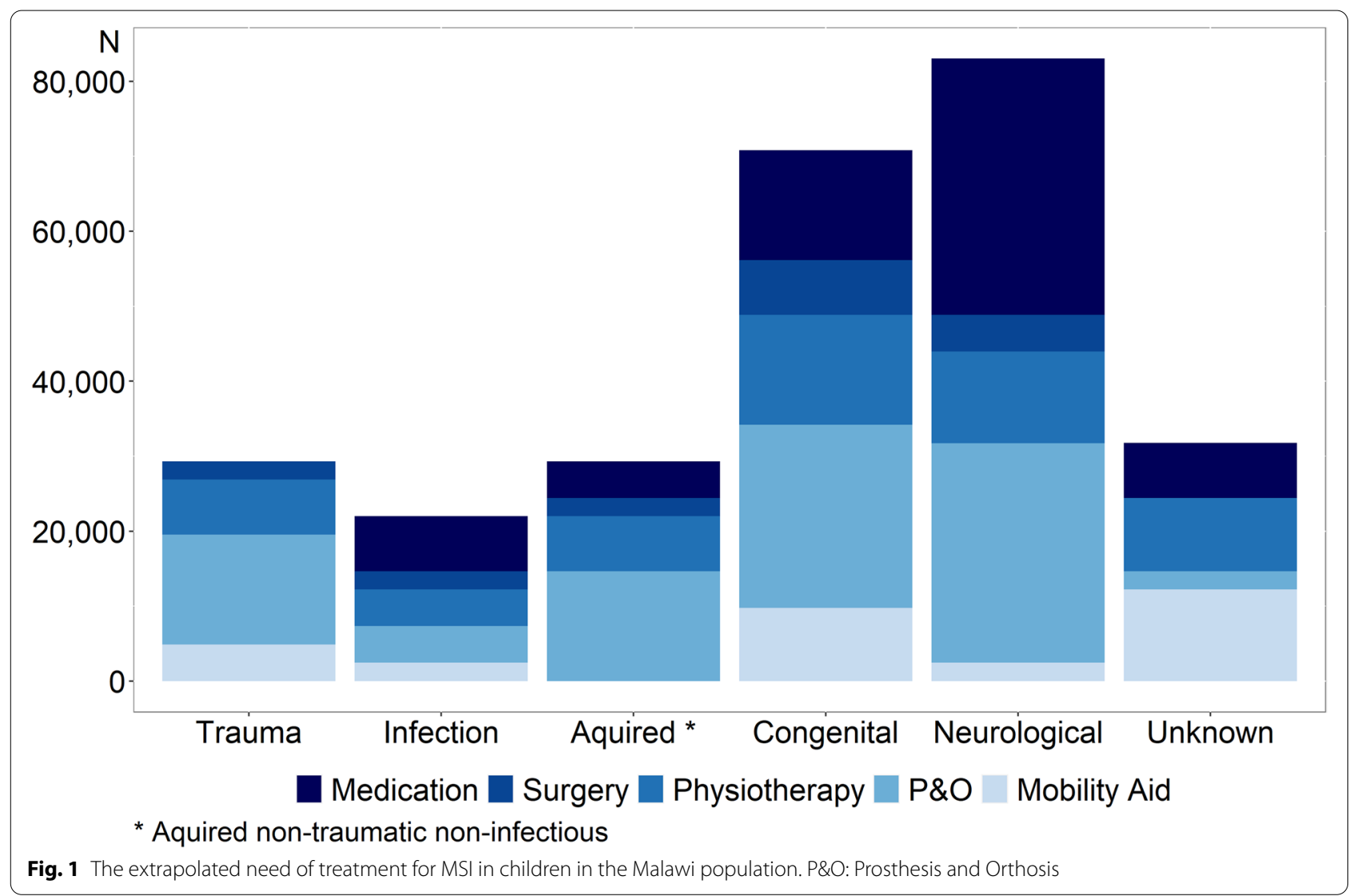

of conditions that needed complex investigations. Other limitations were that data on other aspects, such as previous treatment received (some participants were unable to recall their previous treatment) and locations were not collected, and our demographic data were limited. Due to long travel distances in some areas, the call back at a few households where people were unavailable at the initial visit was not achieved. Extrapolations are always associated with some uncertainty and we have been careful to give $95 \%$ confidence limits. The study also had several strengths. The response rate of over $96 \%$ is very good for a survey in our setting, with the added strength that the study was a nationwide survey with a cluster randomized design to obtain a representative sample of children aged 16 years and younger. The fact that most diagnoses and surgical indications were made by one orthopaedic surgeon, also strengthens the findings and implications in our opinion.

In Malawi, a country with limited resources for health, it is unlikely that the unmet needs for treatment among these children will be met fully in near future. However, the estimates provided in this study should be useful in development of policy and for planning of services in Malawi. With $85 \%$ of the Malawian population living in rural areas, access to health and other services may be improved through community-based rehabilitation programs in rural communities. In addition, the development of programs that serve populations at the district level, where needs can be assessed and resources identified, may improve access to preventative services and rehabilitation.

Regarding CP, being one of the most common disabilities among the children, further studies are needed with regard to causes, types, socioeconomic status, education, severity, and other associated medical conditions.

\section{Conclusion}

This survey has uncovered a large burden of MSI among children aged 16 and under in Malawi. Lack of formal education in the family was one factor associated with an increased risk of MSI. The burden of musculoskeletal impairment in Malawi is mostly unattended, revealing a need to scale up prosthetics and orthotics, physical \& occupational therapy, and surgical services in the country. 


\section{Supplementary Information}

The online version contains supplementary material available at https://doi. org/10.1186/s12887-022-03113-8.

Additional file 1. Appendix: Rapid Assessment of Musculoskeletal Impairment (RAM 1 \& RAM 2)

\section{Acknowledgements}

Not applicable.

\section{Authors' contributions}

Conceptualization: LBN, GH, SY. Data curation: LBN, NM, GH, JE, SY. Investigation: LBN, SY. Project administration: LBN. Data analysis: ED, JE. Writing, review and editing: LBN, CV, NM, GH, ED, JE, SY. The author(s) read and approved the final manuscript.

\section{Funding}

This study was funded by Norad through the Norhed programme (data collection).

\section{Availability of data and materials}

The datasets used and/or analyzed during the current study are available from the corresponding author on reasonable request.

\section{Declarations}

\section{Ethics approval and consent to participate}

The approval to conduct the survey was granted by the University of Malawi College of Medicine Research and Ethics Committee (COMREC) and The Regional Committee for Medical and Health Research Ethics (REC West) in Norway. Data collection and handling followed national guidelines in line with the Declaration of Helsinki. Informed consent to participate in the study was obtained from a parent or guardian before examination. Data collectors were allowed to take clinical photographs for teaching and discussion purposes after a verbal informed consent from the parent or guardian.

\section{Consent for publication}

Not applicable.

\section{Competing interests}

The authors declare that they have no competing interests

\section{Author details}

'Department of Surgery, Kamuzu Central Hospital, P.O. Box 149, Lilongwe, Malawi. ${ }^{2}$ Department of Clinical Medicine, University of Bergen, Bergen, Norway. ${ }^{3}$ Centre for International Health, University of Bergen, Bergen, Norway. ${ }^{4}$ The Norwegian Arthroplasty Register, Haukeland University Hospital, Bergen, Norway. ${ }^{5}$ Department of Orthopaedic Surgery, The Norwegian Arthroplasty Register Haukeland University Hospital, 5021 Bergen, Norway. ${ }^{6}$ Department of Orthopaedic Surgery, Haukeland University Hospital, 5021 Bergen, Norway. ${ }^{7}$ Department of Surgery, College of Medicine, University of Malawi, Blantyre, Malawi. ${ }^{8}$ School of Medicine, Flinders University, Adelaide, Australia.

Received: 14 April 2021 Accepted: 5 January 2022

Published online: 28 January 2022

\section{References}

1. Bethge M, von Groote P, Giustini A, Gutenbrunner C. The World Report on Disability: a challenge for rehabilitation medicine. Am J Phys Med Rehabil. 2014;93(1Suppl1):S4-11. https://doi.org/10.1097/PHM.0000000000 000016.

2. World Health Organization \& World Bank. World report on disability. Geneva: Switzerland World Health Organization; 2011.

3. Banza L, Gallaher J, Dybvik E, Charles A, Hallan G, Gjertsen JE, et al. The rise in road traffic injuries in Lilongwe, Malawi. A snapshot of the growing epidemic of trauma in low income countries. Int J Surg Open. 2018;10:1e6.
4. World Bank Country and Lending Groups; 2017. Available at: https:// datahelpdesk.worldbank.org/knowledgebase/articles/906519-worldbank-country-and-lending-groups. Accessed 19 Sept 2017.

5. Kuper H, Monteath-van Dok A, Wing K, Danquah L, Evans J, Zuurmond M, et al. The impact of disability on the lives of children; cross-sectional data including 8,900 children with disabilities and 898,834 children without disabilities across 30 countries. Plos One. 2014;9(9):e107300.

6. Atijosan O, Rischewski D, Simms V, Kuper H, Lavy C, Foster A, et al. Poverty and musculoskeletal impairment in Rwanda. https://doi.org/10.1016/j. trstmh.2008.02.023.

7. Woolf A, Pfleger B. Burden of major musculoskeletal conditions. Bull World Health Organ. 2003;81:646-56.

8. Reshamwalla S, Gobeze A, Ghosh S, Crimes C, Lavy C. Snapshot of surgical activity in rural Ethiopia: is enough being done? World I Surg. 2012;36:1049-55.

9. Atijosan O, Rischewski D, Simms V, Kuper H, Lavy C. the orthopaedic needs of children in Rwanda: results from a national survey and orthopaedic service implications. J Pediatr Orthop. 2009;29(8):948-51.

10. Tataryn M, Polack S, Chokotho L, Mulwafu W, Kayange P, Banks LM, et al. Childhood disability in Malawi: a population based assessment using the key informant method. BMC Pediatr. 2017;17(1):198. https://doi.org/10. 1186/s12887-017-0948-Z.

11. Alavi Y, Jumbe V, Hartley S, Smith S, Lamping D, Muhit M, et al. Indignity, exclusion, pain and hunger: the impact of musculoskeletal impairments in the lives of children in Malawi. Disabil Rehabil. 2012;34(20):1736-46. https://doi.org/10.3109/09638288.2012.662260.

12. Ngoie LB, Dybvik E, Hallan G, Gjertsen JE, Mkandawire N, Varela C, et al. Prevalence, causes and impact of musculoskeletal impairment in Malawi: A national cluster randomized survey. PLoS ONE. 2021;16(1):e0243536. https://doi.org/10.1371/journal.pone.0243536.

13. Chopra A. COPCORD — an unrecognised fountainhead of community rheumatology in developing countries. J Rheumatol. 2004;31:2320-1.

14. World Health Organisation. International classification of functioning disability and health. Geneva:WHO; 2001.

15. Atijosan O, Kuper H, Rischewski D, Simms V, Lavy C. Musculoskeletal impairment survey in Rwanda: design of survey tool, survey methodology, and results of pilot study (a cross sectional survey). BMC Musculoskelet Disord. 2007:8:30.

16. Smythe T, Mactaggart I, Kuper H, Oye J, Sieyen NC, Lavy C, et al. Prevalence and causes of musculoskeletal impairment in Fundong District, North-West Cameroon: results of a population-based survey. Trop Med Int Health. 2017:22(11):1385-93.

17. Akinyoola AL, Adegbehingbe $1 \mathrm{OO}$, Ogundele OJ. Factors influencing the outcome of elective paediatric orthopaedic operations in Ile-Ife, Nigeria. Tanzania J Health Res. 2008;10(2):68-72.

18. Bax M, Goldstein M, Rosenbaum P, et al. Proposed definition and classification of cerebral palsy, April 2005. Dev Med Child Neurol. 2005;47:571-6.

19. Donald KA, Samia P, Kakooza-Mwesige A, Bearden D. Pediatric cerebral palsy in Africa: a systematic review. Semin Pediatr Neurol. 2014;21:30-5.

20. Monokwane B, et al. Risk Factors for Cerebral Palsy in Children in Botswana. Pediatr Neurol. 2017;77:73-7 A.L.

21. OECD. New ways of Adressing partial work capacity. In: OECD thematic Reiview on sickness, disability and work Isues, paper and Progress report; 2007. www.oecd.org/els/disability.

\section{Publisher's Note}

Springer Nature remains neutral with regard to jurisdictional claims in published maps and institutional affiliations. 